\title{
Pattern occurrences in $k$-ary words revisited: a few new and old observations
}

\author{
Toufik Mansour* Reza Rastegar ${ }^{\dagger}$
}

December 22, 2022

\begin{abstract}
In this paper, we study the pattern occurrence in $k$-ary words. We prove an explicit upper bound on the number of $k$-ary words avoiding any given pattern using a random walk argument. Additionally, we reproduce one already known result on the exponential rate of growth of pattern occurrence in words and establish a simple connection among pattern occurrences in permutations and $k$-ary words. A simple yet interesting consequence of this connection is that the Wilfequivalence of two patterns in words implies their Wilf-equivalence in permutations.
\end{abstract}

MSC2010: Primary 05A05, 05A16, 05C81.

Keywords: pattern avoidance and occurrence, $k$-ary words, permutations, random walk.

\section{Introduction}

The enumeration of pattern occurrences in discrete sequences has been a very active area of research in the last three decades; see for instance the

\footnotetext{
*Department of Mathematics, University of Haifa, 199 Abba Khoushy Ave, 3498838 Haifa, Israel;

e-mail: tmansour@univ.haifa.ac.il

${ }^{\dagger}$ Occidental Petroleum Corporation, Houston, TX 77046 and Departments of Mathematics and Engineering, University of Tulsa, OK 74104, USA - Adjunct Professor; e-mail: reza_rastegar2@oxy.com
} 
monographs [2, 8]. Although, in the beginning, the main focus was mainly on pattern occurrence in permutations, other classes of sequences including $k$-ary words [1, 4, 12] has been also the subject of further research. The main theme of this paper is also the enumeration of pattern occurrences in $k$-ary words.

To state our results we first define a few notations and concepts. Let $\mathbb{N}:=\{1,2,3, \ldots\}$ and $\mathbb{N}_{0}$ denote, respectively, the set of natural numbers and the set of non-negative integers; that is $\mathbb{N}_{0}=\mathbb{N} \cup\{0\}$. For a given set $A$, $\# A$ is the cardinality of $A$. A word $w$ is a sequence $w:=w_{1} \cdots w_{n}$, where $w_{i}$ is the $i$-the entry of $w$ and is chosen from an arbitrary set of letters also known as alphabet. For any given $k \in \mathbb{N}$, we denote the set $\{1,2, \cdots k\}$ by $[k]$. We adopt the convention that $[k]^{0}=\{\epsilon\}$, where $\epsilon$ is an empty word. A $k$-ary word of length $n$ is an element of $[k]^{n}, n \in \mathbb{N}$. A pattern is any distinguished word chosen from $\cup_{\ell}[k]^{\ell}$ containing letters in $[k]$. Let us now fix integers $k \in \mathbb{N}, \ell \geq 2$, and a pattern $v$ in $[k]^{\ell}$. These parameters are considered to be given and fixed throughout the rest of the paper. An important characteristic of the pattern is its number of distinct letters. We will denote this by $d$. For instance, if $v=33415$, then $\ell=5$ and $d=4$. For an arbitrary word $w$ with length $n \geq \ell$, an occurrence of the pattern $v$ in $w$ is a sequence of $\ell$ indexes $1 \leq j_{1}<j_{2}<\cdots<j_{\ell} \leq n$ such that the subsequence $w_{j_{1}} \cdots w_{j_{\ell}}$ is order-isomorphic to the word $v$, that is

$$
w_{j_{p}}<w_{j_{q}} \Longleftrightarrow v_{p}<v_{q} \quad \forall 1 \leq p, q \leq \ell
$$

and

$$
w_{j_{p}}=w_{j_{q}} \Longleftrightarrow v_{p}=v_{q} \quad \forall 1 \leq p, q \leq \ell .
$$

For any arbitrary word $w$ we denote by $\operatorname{occ}_{v}(w)$ the number of occurrences of $v$ in $w$. For instance, if $v$ is the inversion 21 and $w=35239$, then there are three occurrences, those are $w_{1} w_{3}=32, w_{2} w_{3}=52$, and $w_{2} w_{4}=53$, and consequently $\operatorname{occ}_{v}(w)=3$. We say that a word $w$ contains the pattern $v$ exactly $r$ times, $r \in \mathbb{N}_{0}$, if $\operatorname{occ}_{v}(w)=r$. By the occurrence subsequence of $w$, we refer to the minimal length subsequence of the word $w$ containing all the $r$ occurrences of $v$. For instance, in the previous example, the occurrence subsequence are the subsequence 3523 . For a set of words $A$ and $r \in \mathbb{N}_{0}$, we denote by $F_{r}^{v}(A)$ the set of words in $A$ each of which contains $v$ exactly $r$ times. That is,

$$
F_{r}^{v}(A)=\left\{w \in A: \operatorname{occ}_{v}(w)=r\right\}
$$


We use $f_{r}^{v}(A)$ to refer to $\# F_{r}^{v}(A)$. In the case of $r=0$, we use the term avoidance instead of occurrence.

Our first theorem states

Theorem 1.1. For any pattern $v$ of length $\ell$ with $d$ distinct letters and any $k \in \mathbb{N}$ with $k>d$, we have

(a) $(d-1)^{n} \leq f_{0}^{v}\left([k]^{n}\right) \leq(d-1)^{n} \sum_{i=0}^{\ell\left(\begin{array}{l}k \\ d\end{array}\right)}\left(\begin{array}{c}n \\ i\end{array}\right)\left(\frac{k-d+1}{d-1}\right)^{i}$, whenever $n>\ell\left(\begin{array}{l}k \\ d\end{array}\right)$.

(b) $\lim _{n \rightarrow \infty} f_{r}^{v}\left([k]^{n}\right)^{\frac{1}{n}}=d-1$, whenever $r \in \mathbb{N}_{0}$.

(c) $f_{0}^{v}\left([k]^{n}\right)=\sum_{i=0}^{n} a_{i, k, v}(n)(d-1)^{i}$, where $a_{i, k, v}(n)$ are polynomials in $n$ with integer coefficients possibly depending on $k$ and $v$.

Part (a) gives a new general upper bound on $f_{0}^{v}\left([k]^{n}\right)$ which is a function of $\ell, d, k$, and $n$. Its proof, given in Section 3 , is based on a simple random walk argument over the poset of instances of the pattern $v$ for the alphabet $[k]$. This poset is defined in Section 2 .

The statement of part (b) is not new, however, our proof is. The existence of this limit and its value was first proven for the $r=0$ case, i.e. pattern avoidance, in Theorem 3.2 [4] using transfer matrix method (see section 4.7 of [13]). The result was extended later to the general occurrence case $r \in \mathbb{N}$ in Theorem 2.7 [10] by a similar technique. Our approach for $r=0$ case is similar to that of [4], in the sense that, the number of walks in certain graphs are counted. The advantages of our method in proving the case $r=0$ are however twofold: (1) the poset defined in Section 2 is simpler to construct and more intuitive than those defined in [4, 10. (2) our approach has a generalizable probabilistic flavor; for instance, our results can be extended to other contexts, such as when the words are generated by any finite irreducible aperiodic Markov chain. Our proof of the $r \in \mathbb{N}$ case is entirely different from the transfer matrix method used in [10] and it is done directly by relating the $r \in \mathbb{N}$ case to the $r=0$ case.

Recall that a word $w=w_{1} \cdots w_{n}$ is a permutation if it is simply a rearrangement of the sequence $12 \ldots n$; that is $w \in F_{0}^{11}\left([n]^{n}\right)$. We use $\mathcal{S}_{n}$ to refer to the set of all permutations of length $n$. The celebrated Marcus-Tardos Theorem [11], confirming Stanley-Wilf conjecture for pattern avoidance in $\mathcal{S}_{n}$, states that the number of permutations in $\mathcal{S}_{n}$ avoiding a permutation 
pattern $v \in \mathcal{S}_{d}$ grows at most as fast as an exponential function of $n$; that is, there exists a constant $c_{v}$ where for all $n \in \mathbb{N}$,

$$
f_{0}^{v}\left(\mathcal{S}_{n}\right) \leq c_{v}^{n}
$$

Around the time this conjecture was settled, Brändén and Mansour [4] conjectured the plausibility of equivalence of Marcus-Tardos Theorem for $\mathcal{S}_{n}$ with a similar result for $[n]^{n}$. More precisely, they conjectured that there are $c_{v}$ and $g_{v}$ where for all $n \in \mathbb{N}$ the following statement holds:

$$
f_{0}^{v}\left(\mathcal{S}_{n}\right)<c_{v}^{n} \quad \text { if and only if } f_{0}^{v}\left([n]^{n}\right)<g_{v}^{n} .
$$

By a reformulation of the pattern avoidance in the language of graph theory, Corollary $2.2[9]$ settled the existence of $g_{v}>0$ where $f_{0}^{v}\left([n]^{n}\right)<g_{v}^{n}$ for all $n \in \mathbb{N}$. However, yet there is no direct explanation on why (2) actually holds and whether something nontrivial could be said about the relationship between the optimal values of $c_{v}$ and $g_{v}$. That being said, our motivation behind Theorem 1.2 bellow was originally to establish some connection between $f_{0}^{v}\left([n]^{n}\right)$ and $f_{0}^{v}\left(\mathcal{S}_{0}^{v}\right)$ (see also the inequality (19) $)$ :

Theorem 1.2. Let $v$ be any pattern. Then, for $r \in \mathbb{N}_{0}$,

$$
f_{r}^{v}\left(\mathcal{S}_{n}\right)=\sum_{k=1}^{n}(-1)^{n-k}\left(\begin{array}{l}
n \\
k
\end{array}\right) f_{r}^{v}\left([k]^{n}\right)
$$

Furthermore, let $S_{r}^{v}(x)$ and $W_{r, k}^{v}(x)$ be the exponential generating functions of $f_{r}^{v}\left(\mathcal{S}_{n}\right)$ and $f_{r}^{v}\left([k]^{n}\right)$; those are,

$$
S_{r}^{v}(x)=\sum_{n=1}^{\infty} \frac{f_{r}^{v}\left(\mathcal{S}_{n}\right)}{n !} x^{n} \quad \text { and } \quad W_{r, k}^{v}(x)=\sum_{n=1}^{\infty} \frac{f_{r}^{v}\left([k]^{n}\right)}{n !} x^{n}, \quad k \in \mathbb{N} .
$$

Then

$$
S_{r}^{v}(x)=\sum_{k=1}^{\infty} \frac{(-x)^{k}}{k !} \frac{\partial^{k}}{\partial x^{k}} W_{r, k}^{v}(-x)
$$

We remark that (3) is indeed correct if we replace the pattern $v$ with a set of patterns. We end this section by stating an interesting immediate consequence of Theorem 1.2: 
Corollary 1.3. For any two patterns $v_{1}$ and $v_{2}$, if $f_{0}^{v_{1}}\left([k]^{n}\right)=f_{0}^{v_{2}}\left([k]^{n}\right)$ for all $k$ and $n$, then $f_{0}^{v_{1}}\left(\mathcal{S}_{n}\right)=f_{0}^{v_{2}}\left(\mathcal{S}_{n}\right)$ for all $n$. The inverse statement does not hold.

This implies that Wilf-equivalence in words implies Wilf-equivalence in permutations. An example for the "if" statement is the following: it was shown in [5] that

$f_{0}^{123}\left([k]^{n}\right)=f_{0}^{132}\left([k]^{n}\right)=\delta_{k, 1}+2^{n-2(k-2)} \sum_{j=0}^{k-2}\left(\begin{array}{c}n+2 j \\ n\end{array}\right)\left(\sum_{m=j}^{k-2} \frac{1}{m+1}\left(\begin{array}{c}2 m \\ m\end{array}\right)\left(\begin{array}{c}2 k-2 m \\ k-m\end{array}\right)\right)$

where $\delta_{k, 1}=1$ if $k=1$, and is zero otherwise. Therefore, the corollary implies $f_{0}^{123}\left(\mathcal{S}_{n}\right)=f_{0}^{132}\left(\mathcal{S}_{n}\right)$. This is indeed known to hold by a direct calculation [2], showing

$$
f_{0}^{123}\left(\mathcal{S}_{n}\right)=f_{0}^{132}\left(\mathcal{S}_{n}\right)=\frac{1}{n+1}\left(\begin{array}{c}
2 n \\
n
\end{array}\right) .
$$

To see the inverse statement in the corollary, take for example $v_{1}=1324$ and $v_{2}=2413$. As it is shown by Bóna [3] $f_{0}^{v_{1}}\left(S_{n}\right)=f_{0}^{v_{2}}\left(S_{n}\right)$; however, we know from [7], $f_{0}^{v_{1}}\left([k]^{n}\right)$ and $f_{0}^{v_{2}}\left([k]^{n}\right)$ are not same for all $k$ and $n$.

\section{Instances of a pattern}

In this section, we first define a partially ordered set (poset) based on the instances of a given pattern $v$ in the alphabet $[k]$ and discuss a few of its basic properties. To that end, for a given pattern $v$ of length $\ell$ with $d$ distinct letters, we define $\Phi_{k}(v)$ to be the set of all words $w \in[k]^{\ell}$ for which $\operatorname{occ}_{v}(w)=1$. The size of this set, $\phi_{k}(v):=\# \Phi_{k}(v)$, is $\left(\begin{array}{l}k \\ d\end{array}\right)$. For instance,

$$
\Phi_{4}(123)=\{123,124,134,234\} .
$$

To decide whether a given word $w$ is in $F_{0}^{v}\left([k]^{n}\right)$ or not, we can advise a simple avoidance detection algorithm, as follows: it reads the letters in $w$ from left to right and updates a state vector reflecting the prefixes of the pattern instances in $\Phi_{k}(v)$ that have been encountered after any letter of $w$ is consumed. Clearly, the word $w$ avoids the pattern $v$, as long as, none of the state vector entries coincides with any element in $\Phi_{k}(v)$ by the time that 
the rightmost letter of $w$ is processed. Intuitively, this is a walk on the space of "feasible" state vectors. To formalize this idea, we equip $\Phi_{k}(v)$ with an order $<_{\circ}$ : for any pair $u, t \in \Phi_{k}(v)$, we say $u<_{\circ} t$, if and only if, $u_{i}<_{\circ} t_{i}$ for the first index $1 \leq i \leq \ell$ where $u_{i} \neq t_{i}$. For example, elements in $\Phi_{4}(123)$ are ordered as

$$
123<_{\circ} 124<_{\circ} 134<_{\circ} 234
$$

For each $u \in \Phi_{k}(v)$, set $\Xi(u)$ to be the set of all prefixes of $u$ including the empty word $\epsilon$ and $u$ itself. For instance,

$$
\Xi(1245)=\{\epsilon, 1,12,124,1245\}
$$

Regardless of the value of $u \in \Phi_{k}(v)$, the size of $\Xi(u)$ is always $\ell+1$. Next, set $\xi^{\epsilon}:=(\epsilon, \epsilon, \cdots, \epsilon)$ to be the null state vector and define

$$
\Xi_{k}(v):=\left\{\xi:=\left(\xi_{u}\right)_{u \in \Phi_{k}(v)} \mid \xi_{u} \in \Xi(u), \forall u \in \Phi_{k}(v)\right\} .
$$

The indexes of the entries of any $\xi \in \Xi_{k}(v)$ s are ordered according to $<_{0}$.

For any two given states $\xi, \eta \in \Xi_{k}(v)$, we say $\xi$ is extendable to $\eta$ in one step if there are a non-empty subset $A \in \Phi_{k}(v)$ and a unique $i \in[k]$ for which $\eta_{t}=\xi_{t} i$ (concatenation of the word $\xi_{t}$ and letter $i$ ) for $t \in A$, and $\eta_{t}=\xi_{t}$, otherwise. We use the notation $\nu=\xi i$ in this case. Additionally, we define an order $<_{\diamond}$ on $\Xi_{k}(v)$ : for any pair of $\eta, \xi \in \Xi_{k}(v)$, we say $\xi<_{\diamond} \eta$, if and only if, $\xi$ is extendable to $\eta$ in $s$ steps for some finite $s \in \mathbb{N}$; that is, $\eta=\xi i_{1} \ldots i_{s}:=\left(\ldots\left(\left(\xi i_{1}\right) \ldots\right) i_{s}\right.$ for a finite sequence of letters $i_{1}, \cdots, i_{s} \in[k]$. Let $\mathcal{E}_{k}(v)$ be the set

$$
\mathcal{E}_{k}(v):=\left\{\xi^{\epsilon}\right\} \cup\left\{\xi \in \Xi_{k}(v), \xi^{\epsilon}<_{\diamond} \xi\right\} .
$$

Then, each $\xi \in \mathcal{E}_{k}(v)$ can be obtained by a finite extension of $\xi^{\epsilon}$; that is, $\xi^{\epsilon}$ is extendable in some finite number of steps to $\xi$. Define

$$
\mathcal{O}_{k}(v):=\left\{\xi \in \mathcal{E}_{k}(v) \mid \xi_{u}=u \text { for some } u \in \Phi_{k}(v)\right\}
$$

and set $\mathcal{V}_{k}(v):=\mathcal{E}_{k}(v) \backslash \mathcal{O}_{k}(v)$. It is clear that $\mathcal{V}_{k}(v)$ is a poset with respect to $<_{\diamond}$. See Figures 1 1 3 for Hasse diagram of several examples of this poset with different patterns and the alphabet $[4]=\{1,2,3,4\}$. For each $\xi \in \mathcal{V}_{k}(v)$, let $L_{v, k}(\xi)$ be the subset of alphabet $[k]$ whose elements extend $\xi$ in $\mathcal{V}_{k}(v) \cup \mathcal{O}_{k}(v)$. 


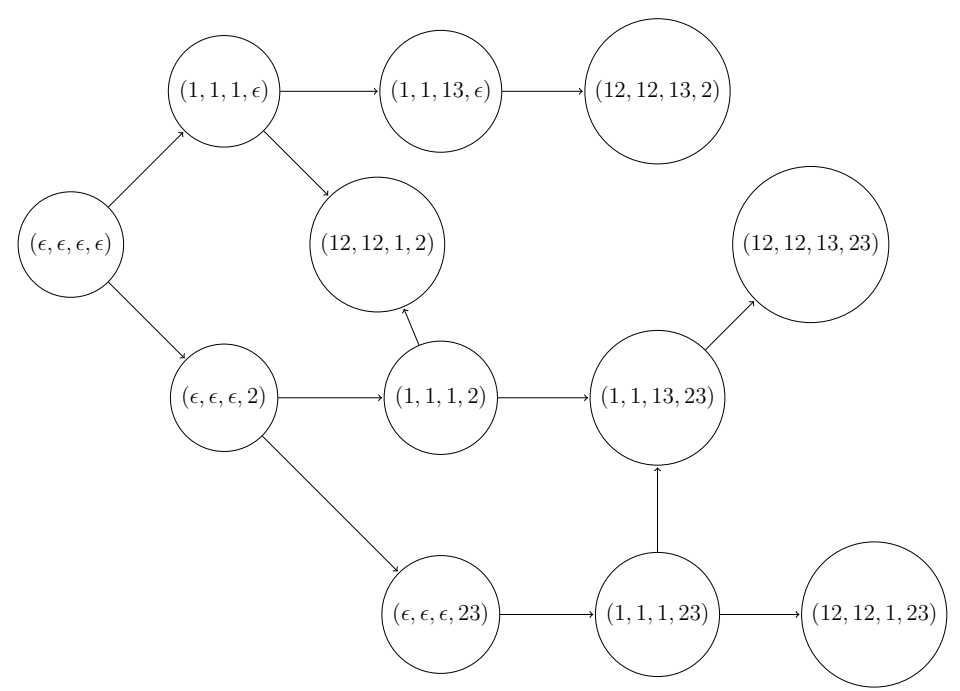

Figure 1: The Hasse diagram for the poset $\mathcal{V}_{k}(v)$ with $v=123$ and $k=4$.

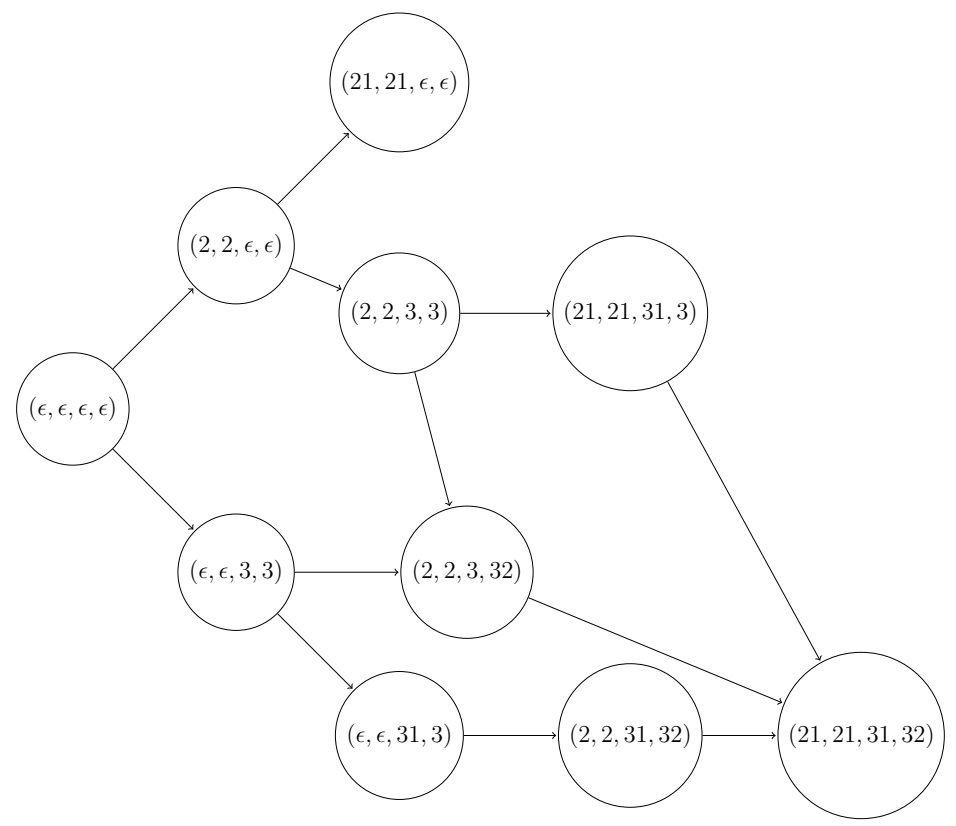

Figure 2: The Hasse diagram for the poset $\mathcal{V}_{k}(v)$ with $v=213$ and $k=4$. 


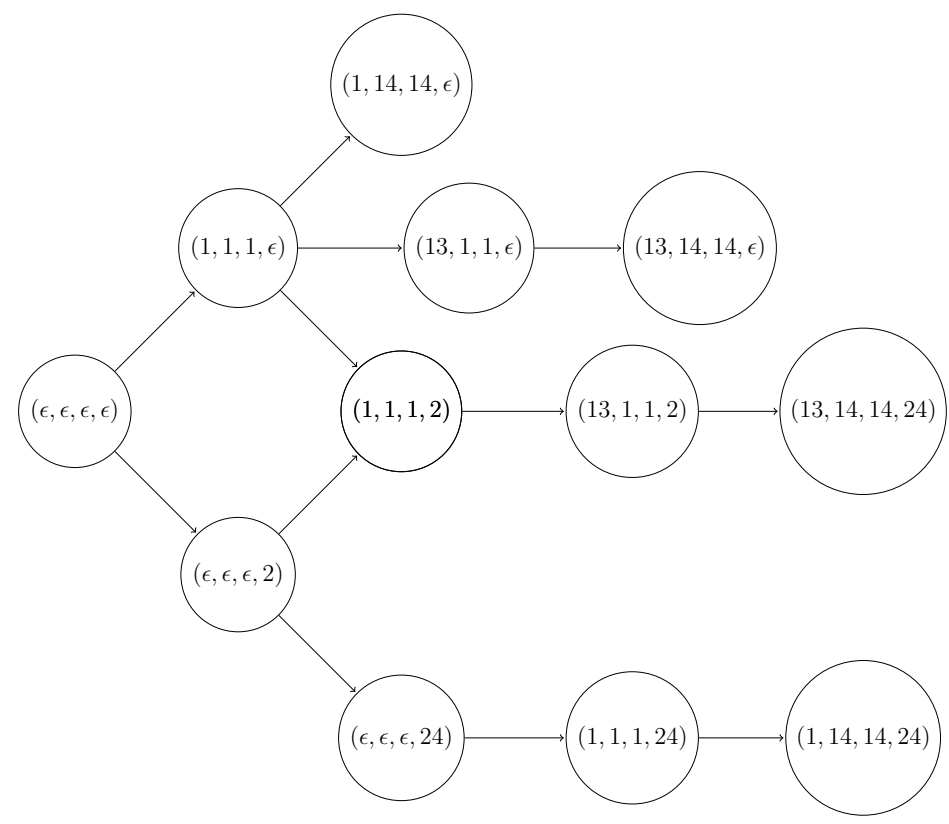

Figure 3: The Hasse diagram for the poset $\mathcal{V}_{k}(v)$ with $v=132$ and $k=4$.

Take $v, w$ as the input data.

Let $n$ be the length of $w$, and $k$ be the number of distinct letters in $w$.

Set $\xi:=\xi^{\epsilon}$ and $i=1$.

Compute $\Phi_{k}(v), \mathcal{E}_{k}(v), \mathcal{V}_{k}(v)$, and $L_{v, k}($.$) .$

while $i \leq n$ do

if $w_{i} \in L_{v, k}(\xi)$ then

Set $\xi$ to $\xi w_{i}$

end if

Increase $i$ by one.

end while

if $\xi \in \mathcal{V}_{k}(v)$ then

return $w$ avoids $v$

else

return $w$ includes $v$

end if

Figure 4: The pseudo-code of the avoidance detection algorithm.

With these notations in hand, the avoidance detection algorithm de- 
scribed informally in the beginning of this section is formalized as shown in Figure 4. In the rest of this section, we provide some basic information on two important objects used by this algorithm; namely $\mathcal{V}_{k}(v)$ and $L_{v, k}($.$) .$ The first result gives an upper bound on the depth of the poset $\mathcal{V}_{k}(v)$ :

Lemma 2.1. For any pattern $v$, let $h_{k}(v)$ be the size of the maximum chain of $\mathcal{V}_{k}(v)$. Then,

$$
h_{k}(v) \leq\left(\begin{array}{l}
k \\
d
\end{array}\right) \ell
$$

Proof. Recall that every element of $\mathcal{V}_{k}(v)$ has $\left(\begin{array}{l}k \\ d\end{array}\right)$ entries, where for each $u \in \Phi_{k}(v)$, the corresponding entry can only take its values from the set of $\ell$ distinct values

$$
\left\{\epsilon, u_{1}, u_{1} u_{2}, \cdots, u_{1} \ldots u_{\ell-1}\right\}
$$

Hence the result follows.

We end this section with the following observation.

Lemma 2.2. Suppose $v$ is any pattern with $d$ distinct letters. For any $\xi \in \mathcal{V}_{k}(v)$, we have

$$
\# L_{v, k}(\xi) \geq k-d+1 \text {. }
$$

Proof. The basic idea of the proof is that with the alphabet $[k]$ and the pattern $v$ made of $d$ distinct letters, we can bound $\# L_{v, k}($.$) from bellow in$ terms of either $\# L_{v^{\prime}, k-1}($.$) or \# L_{v, k-1}($.$) for a certain pattern v^{\prime}$ with $d-1$ distinct letters. Hence, an inductive argument with respect to $k$ and $d$ would be a natural option to prove the statement. First, note that for any pattern $v$ with $d$ distinct letters, we have

$$
\begin{aligned}
\# L_{v, d}(\xi) & =1, \text { for all } \xi \in \mathcal{V}_{d}(v), \\
L_{v, k}\left(\xi^{\epsilon}\right) & =\left\{v_{1}, \cdots, k-d+v_{1}\right\}, \quad \# L_{v, k}\left(\xi^{\epsilon}\right)=k-d+1 .
\end{aligned}
$$

Furthermore, for $v$ with $d=1$

$$
\# L_{v, k}(\xi)=k \text { for all } \xi \in \mathcal{V}_{k}(v) \text {. }
$$

Now, we state our induction hypothesis: 
For some fixed $d, k \in \mathbb{N}$ with $k \geq d$, and any pattern $v$ with $d_{0} \leq d$ distinct letters, if $\xi \in \mathcal{V}_{k}(v)$, then we have $\# L_{v, k_{0}}(\xi) \geq k_{0}-d_{0}+1$ with $d_{0} \leq k_{0} \leq k$.

Finally, we complete the induction argument by showing that for these $k$ and $d$,

$$
\begin{gathered}
\# L_{v, k+1}(\rho) \geq k-d+2, \text { for all } \rho \in \mathcal{V}_{k+1}(v) \\
\# L_{v^{\prime \prime}, k}(\rho) \geq k-d, \text { for all } \rho \in \mathcal{V}_{k}\left(v^{\prime \prime}\right)
\end{gathered}
$$

where $v^{\prime \prime}$ is a pattern obtained by inserting one or more copies of the letter $d+1$ into any arbitrary position(s) in $v$.

To that end, let $v$ be any pattern of $d$ distinct letters. If $\rho=\rho^{\epsilon}:=(\epsilon, \cdots, \epsilon) \in \mathcal{V}_{k+1}(v)$, then by (8) we are done. Otherwise, we consider two cases for any other $\rho \in \mathcal{V}_{k+1}(v):$

1. if $k+1 \in L_{v, k+1}(\rho)$, then define $\xi$ to be the set of $\rho_{u}$ s where $u$ does not have the letter $k+1$. Clearly $\xi \in \mathcal{V}_{k}(v), k+1 \notin L_{v, k}(\xi)$,

$$
L_{v, k}(\xi) \cup\{k+1\} \subset L_{v, k+1}(\rho) \text {, and } \# L_{v, k}(\xi)+1 \leq \# L_{v, k+1}(\rho) .
$$

Here, $\xi$ should be understood as the vector of elements in the set ordered by $<_{0}$.

2. if $k+1 \notin L_{v, k+1}(\rho)$, then define $v^{\prime}$ to be a pattern with $d-1$ distinct letters; obtained by dropping the letter $d$ everywhere in $v$. Also, define $\xi$ to be the set of $\nu_{u}$ s after dropping the letter $k+1$ (if any) where $u$ has the letter $k+1$. In this case, we have $\xi \in \mathcal{V}_{k}\left(v^{\prime}\right)$,

$$
L_{v^{\prime}, k}(\xi) \subset L_{v, k+1}(\rho) \text {, and } \# L_{v^{\prime}, k}(\xi) \leq \# L_{v, k+1}(\rho) .
$$

These two cases along with the induction hypothesis yield (9). Inequality (10) can also be proven using an identical argument. This completes the proof.

\section{Proof of Theorem 1.1}

This section is devoted to the proof of Theorem 1.1. We start with a few definitions. 
Let $\mathbb{P}$ be a probability law that induces the uniform distribution on $[k]$. For technical convenience, we enlarge the probability space of $\mathbb{P}$ such that it contains all random variables used in the following. Let $(X(n))_{n \in \mathbb{N}}$ be an i.i.d. sequence of random variables where $X(1)$ is distributed as a uniform variable on $[k]$; that is,

$$
\mathbb{P}(X(n)=i)=\frac{1}{k} \quad \forall i \in[k], \quad n \in \mathbb{N} .
$$

Set the sequence $Z:=(Z(n))_{n \in \mathbb{N}_{0}}$ where $Z(0):=\xi^{\epsilon}$ and

$$
Z(n+1)=\left(E_{u}\left(Z_{u}(n), X(n+1)\right)\right)_{u \in \Phi_{v}(k)},
$$

where $E_{u}(.,):. \Xi(u) \times[k] \rightarrow \Xi(u)$ is defined by

$$
E_{u}\left(\xi_{u}, x\right)= \begin{cases}\xi_{u} & \text { if } \xi_{u} x \notin \Xi(u) \\ \xi_{u} x & \text { otherwise }\end{cases}
$$

for $\xi_{u} \in \Xi_{u}, x \in[k]$, and $u \in \Phi_{k}(v)$.

Note that $Z$ is a Markov chain on $\mathcal{E}_{k}(v)$ and we use $P_{v, k}$, or simply $P$, to refer to the transition matrix of $Z(n)$ defined as follows. For any two given states $\xi, \nu \in \mathcal{E}_{k}(v)$,

$$
P(\xi, \nu)=P_{v, k}(\xi, \nu):= \begin{cases}\frac{1}{k} & \nu=\xi i \text { for some } i \in[k] \\ 1-\frac{\# L_{v, k}(\xi)}{k} & \nu=\xi \\ 0 & \text { otherwise. }\end{cases}
$$

By Lemma 2.2, this yields, for $\xi \in \mathcal{V}_{k}(\xi)$,

$$
P\left(\xi^{\epsilon}, \xi^{\epsilon}\right)=\frac{d-1}{k}, \quad \text { and } \quad P(\xi, \xi) \leq \frac{d-1}{k} .
$$

To summarize, $P$ may be written as an upper triangular matrix in which the values of all non-zero non-diagonal entries are $1 / k$ and the diagonal entries are bounded above by $(d-1) / k$.

We are now ready to give the

Proof of Theorem 1.1-(a) and (c). By the avoidance detection algorithm (Figure (4) and the definition of $Z(n), F_{0}^{v}\left([k]^{n}\right)$ can be described in terms of 
the set of realizations of the Markov chain $Z$ up to the time $n$ in which $Z(0), Z(1), \cdots, Z(n) \in \mathcal{V}_{k}(v)$. That being said, we may write

$$
f_{0}^{v}\left([k]^{n}\right)=k^{n} \sum_{\xi \in \mathcal{V}_{k}(v)} P^{(n)}\left(\xi^{\epsilon}, \xi\right),
$$

where $P^{(n)}$ is the $n$-fold transition probability matrix of the Markov chain $Z$. Given that all nonzero entries of $P$ are of form $\frac{i}{k}$, with $1 \leq i \leq d-1$, and $P\left(\xi^{\epsilon}, \xi^{\epsilon}\right)=\frac{d-1}{k}$, the proof of the part (c) follows.

We now prove the part (a). To that end, the lower bound is simply obtained by the observation that any word in $[d-1]^{n} \subset[k]^{n}$ avoids $v$. To obtain the upper-bound, first we define a pure birth process $(\tilde{Z}(n))_{n \in \mathbb{N}_{0}} \subset \mathbb{N}^{\mathbb{N}}$, where

$$
\mathbb{P}(\tilde{Z}(0)=0)=1
$$

and

$$
\mathbb{P}(\tilde{Z}(n+1)=i \mid \tilde{Z}(n)=i)=1-\mathbb{P}(\tilde{Z}(n+1)=i+1 \mid \tilde{Z}(n)=i)=\frac{d-1}{k},
$$

for all $n \in \mathbb{N}_{0}$ and $i \in \mathbb{N}$. Clearly, $\tilde{Z}(n+1)-\tilde{Z}(n)$ is a Bernoulli random variable with the success probability $\frac{k-d+1}{k}$. Hence, $\tilde{Z}(n)$ is sum of $n$ Bernoulli random variables of parameter $\frac{k-d+1}{k}$.

Second, we point out that

1. once the process $Z(n)$ leaves a given state $\xi$, it will never come back to $\xi$ again,

2. starting from $\xi^{\epsilon}, Z(n)$ can take maximum $h_{k}(v)$ distinct values before leaving the set $\mathcal{V}_{k}(v)$,

3. by (12), $Z(n) \in \mathcal{V}_{k}(n)$ stays in its current state with maximum probability $(d-1) / k$.

Thus,

$$
\begin{aligned}
& \mathbb{P}\left(Z(n) \in \mathcal{V}_{k}(v)\right) \leq \mathbb{P}\left(\tilde{Z}(n) \leq h_{k}(v)\right) \\
& \quad=\frac{1}{k^{n}} \sum_{i=0}^{h_{k}(v)}\left(\begin{array}{l}
n \\
i
\end{array}\right)(k-d+1)^{i}(d-1)^{n-i} \leq \frac{(d-1)^{n}}{k^{n}} \sum_{i=0}^{\ell\left(\begin{array}{c}
k \\
d
\end{array}\right)}\left(\begin{array}{l}
n \\
i
\end{array}\right)\left(\frac{k-d+1}{d-1}\right)^{i},
\end{aligned}
$$

where for the last inequality we used ([6). Finally, note that $f_{0}^{v}\left([k]^{n}\right)$ is simply $k^{n} \mathbb{P}\left(Z(n) \in \mathcal{V}_{k}(v)\right)$ and hence the proof is complete. 
We conclude this section with the

Proof of Theorem 1.1-(b). The $r=0$ case is an immediate consequence of part (a) since the summation term in the upper bound is a finite polynomial of $n$ :

$$
\begin{aligned}
d-1 & \leq \liminf _{n \rightarrow \infty} f_{0}^{v}\left([k]^{n}\right)^{\frac{1}{n}} \leq \limsup _{n \rightarrow \infty} f_{0}^{v}\left([k]^{n}\right)^{\frac{1}{n}} \\
& \leq(d-1) \limsup _{n \rightarrow \infty}\left(\sum_{i=0}^{\ell\left(\begin{array}{c}
k \\
d
\end{array}\right)}\left(\begin{array}{l}
n \\
i
\end{array}\right)\left(\frac{k-d+1}{d-1}\right)^{i}\right)^{\frac{1}{n}}=d-1 .
\end{aligned}
$$

To prove the result for the general case $r \in \mathbb{N}$, let $f_{r, s}^{v}\left([k]^{n}\right)$ be the number of words in $[k]^{n}$ with exactly $r$ occurrences of the pattern $v$ and the occurrence subsequence of length $s$. Clearly, for $s \notin\{\ell+r-1, \cdots, r \ell-1, r \ell\}$, we have $f_{r, s}^{v}\left([k]^{n}\right)=0$. Hence, $f_{r}^{v}\left([k]^{n}\right)$ can be written as

$$
f_{r}^{v}\left([k]^{n}\right)=\sum_{s=\ell+r-1}^{r \ell} f_{r, s}^{v}\left([k]^{n}\right) .
$$

Since for each $k$-ary word of length $n$ with $r$ occurrences of $v$ and $s$ occurrence subsequence

1. removing the entire occurrence subsequence gives a new word of length $n-s$ that avoids $v$,

2. the occurrence subsequence may happen in any of $\left(\begin{array}{l}n \\ s\end{array}\right)$ locations in the word, and

3. the number of possibilities for the occurrence subsequence is bounded above by $k^{s}$,

then

$$
f_{0}^{v}\left([k]^{n-s}\right) \leq f_{r, s}^{v}\left([k]^{n}\right) \leq k^{s}\left(\begin{array}{l}
n \\
s
\end{array}\right) f_{0}^{v}\left([k]^{n-s}\right) .
$$

Equations (13) and (14) together imply

$$
f_{0}^{v}\left([k]^{n-r \ell}\right) \leq f_{r}^{v}\left([k]^{n}\right)=\sum_{s=\ell+r-1}^{r \ell} f_{r, s}^{v}\left([k]^{n}\right) \leq \sum_{s=\ell+r-1}^{r \ell} k^{s}\left(\begin{array}{l}
n \\
s
\end{array}\right) f_{0}^{v}\left([k]^{n-s}\right) .
$$


Hence, by taking the limit from all the sides of the inequality, we arrive at

$$
\begin{aligned}
& \liminf _{n \rightarrow \infty} f_{0}^{v}\left([k]^{n-r \ell}\right)^{\frac{1}{n}} \leq \liminf _{n \rightarrow \infty} f_{r}^{v}\left([k]^{n}\right)^{\frac{1}{n}} \\
& \quad \leq \limsup _{n \rightarrow \infty} f_{r}^{v}\left([k]^{n}\right)^{\frac{1}{n}} \leq \limsup _{n \rightarrow \infty}\left(\sum_{s=\ell+r-1}^{r \ell} k^{s}\left(\begin{array}{l}
n \\
s
\end{array}\right) f_{0}^{v}\left([k]^{n-s}\right)\right)^{\frac{1}{n}} .
\end{aligned}
$$

Left side is clearly $d-1$ by the $r=0$ case. The right side is also $d-1$ since

1. it is a finite sum with the number of terms independent of $n$, and

2. the coefficients of $f_{0}^{v}\left([k]^{n-s}\right) \mathrm{s}$ are finite degree polynomials in $n$.

The proof of the general case $r \in \mathbb{N}$ is complete.

\section{Proof of Theorem 1.2}

This section is devoted to the the proof of Theorem 1.2 relating the pattern occurrence in $[k]^{n}$ and in $\mathcal{S}_{n}$.

Proof of Theorem 1.Q. (a). Recall (1). Let $Y_{n}(A)$ be the set of all words of length $n$ where the distinct set of letters of each word is exactly the set $A$. It is defined as

$$
Y_{n}(A):=A^{n} \backslash\left(\cup_{e \in A} A_{e}^{n}\right) \quad \text { where } \quad A_{e}:=A \backslash\{e\} .
$$

Observe that this implies

$$
F_{r}^{v}\left(Y_{n}(A)\right)=F_{r}^{v}\left(A^{n}\right) \backslash\left(\cup_{e \in A} F_{r}^{v}\left(A_{e}^{n}\right)\right) .
$$

Additionally, for any non-empty subset $I \subset A$,

$$
\cup_{e \in I} F_{r}^{v}\left(A_{e}^{n}\right)=F_{r}^{v}\left(\cup_{e \in I} A_{e}^{n}\right)
$$

and

$$
\cap_{e \in I} F_{r}^{v}\left(A_{e}^{n}\right)=\cap_{e \in I} F_{r}^{v}\left(A_{e}^{n}\right) .
$$


Then, an application of the inclusion exclusion principle to (15) yields

$$
\begin{aligned}
& f_{r}^{v}\left(Y_{n}(A)\right)=f_{r}^{v}\left(A^{n}\right)-\sum_{I \subset A}(-1)^{n-\# I} f_{r}^{v}\left(\cap_{e \in I} A_{e}^{n}\right) \\
& =\sum_{I \subset A}(-1)^{\# I} f_{r}^{v}\left((A \backslash I)^{n}\right) .
\end{aligned}
$$

Since $f_{0}^{v}\left(A^{n}\right)=f_{0}^{v}\left(B^{n}\right)$ for any $A, B \subset[n]$ where $A$ and $B$ are the same size, for each $a \in \mathbb{N}$, we get

$$
f_{r}^{v}\left(Y_{n}([a])\right)=f_{r}^{v}\left([a]^{n}\right)+\sum_{k=1}^{a-1}(-1)^{k}\left(\begin{array}{l}
a \\
k
\end{array}\right) f_{r}^{v}\left([a-k]^{n}\right) .
$$

Finally, observe that $Y_{n}([n])=\mathcal{S}_{n}$. This completes the proof of (3)).

To prove (44), note that

$$
\frac{\partial^{k}}{\partial x^{k}} W_{r, k}^{v}(-x)=(-1)^{k} \sum_{n=k}^{\infty} \frac{(-x)^{n-k}}{(n-k) !} f_{r}^{v}\left([k]^{n}\right) .
$$

By multiplying the terms in Lemma 1.2 by $x^{n} / n$ !, adding up over natural numbers, and some simplification we have

$$
\begin{aligned}
S_{r}^{v}(x) & =\sum_{n=1}^{\infty} \frac{x^{n}}{n !} \sum_{k=1}^{n}(-1)^{n-k}\left(\begin{array}{l}
n \\
k
\end{array}\right) f_{r}^{v}\left([k]^{n}\right) \\
= & \sum_{k=1}^{\infty} \frac{x^{k}}{k !} \sum_{n=k}^{\infty} \frac{(-x)^{n-k}}{(n-k) !} f_{r}^{v}\left([k]^{n}\right)=\sum_{k=1}^{\infty} \frac{(-x)^{k}}{k !} \frac{\partial^{k}}{\partial x^{k}} W_{r, k}^{v}(-x) .
\end{aligned}
$$

The proof of this part is complete.

It is not hard to see $f_{0}^{12}\left([k]^{n}\right)$ is given by $\left(\begin{array}{c}n+k-1 \\ n\end{array}\right)$ and $f_{0}^{12}\left(\mathcal{S}_{n}\right)=1$. Thus, an application of Theorem 1.2 (a) leads to the following identity

$$
\sum_{k=1}^{n}(-1)^{n-k}\left(\begin{array}{l}
n \\
k
\end{array}\right)\left(\begin{array}{c}
n+k-1 \\
n
\end{array}\right)=1 .
$$

We also point out that Theorem 1.1.(c), together with (3), yields

$$
f_{r}^{v}\left(\mathcal{S}_{n}\right)=\sum_{i=0}^{n}(d-1)^{i}\left(\sum_{k=1}^{n}(-1)^{n-k}\left(\begin{array}{l}
n \\
k
\end{array}\right) a_{i, k, v}(n)\right),
$$


where all $a_{i, k, v}(n)$ s are polynomials in $n$ with integer coefficients possibly depending on $k, v$, and $d$. Identity (16) may provide some direct informal justification about why $c_{v}$ is an exponential function of $d$ for certain patterns. This statement was formally established by Fox [6] in a recent paper, where the proof relies on a refinement and extension of the framework developed in [11.

We finally end this section with the following observation. Note that for any set $A$, the partition $A^{n}=\cup_{I \subseteq A} Y_{n}(A \backslash I)$ yields

$$
f_{0}^{v}\left(A^{n}\right)=\sum_{I \subseteq A} f_{0}^{v}\left(Y_{n}(A \backslash I)\right) .
$$

In addition, we have

$$
f_{0}^{v}\left(Y_{n}(A \backslash I)\right) \leq\left(\begin{array}{c}
n \\
i
\end{array}\right) f_{0}^{v}\left([i]^{n-i}\right) f_{0}^{v}\left(\mathcal{S}_{i}\right),
$$

since we may decompose each $w \in F_{0}^{v}\left([n]^{n}\right)$ into two words one of which is of length $i$ with $i$ distinct letters that avoids $v$ (the number of such words is $f_{0}^{v}\left(\mathcal{S}_{i}\right)$ ) and the other is in $[i]^{n-i}$ avoiding $v$ (the number of such words is $\left.f_{0}^{v}\left([i]^{n-i}\right)\right)$. These two words can be combined in $\left(\begin{array}{l}n \\ i\end{array}\right)$ ways. Hence, by setting $A=[n]$ in (17) and (18), we arrive at

$$
f_{0}^{v}\left([n]^{n}\right) \leq \sum_{i=1}^{n}\left(\begin{array}{c}
n \\
i
\end{array}\right)^{2} f_{0}^{v}\left([i]^{n-i}\right) f_{0}^{v}\left(\mathcal{S}_{i}\right) .
$$

\section{Acknowledgement}

R.R. would like to thank Alex Roitershtein for many fruitful conversations on the pattern avoidance and occurrence. We also would to thank Zachary Hunter for pointing out a gap in the proof of Theorem 1.1 in an earlier draft of this paper.

\section{References}

[1] N. Alon, E. Friedgut, On the number of permutations avoiding a given pattern, J. Combin. Theory Ser. A 89:1 (2000), 133-140. 
[2] M. Bóna, Combinatorics of Permutations, Chapman \& Hall/CRC, Boca Raton, Second Edition, 2012.

[3] M. Bóna, Exact enumeration of 1342-avoiding permutations: a close link with labeled trees and planar maps, J. of Combin. Theory Ser A 80:2 (1997), 257-272.

[4] P. Brändén, T. Mansour, Finite automata and pattern avoidance in words, J. Combin. Theory Ser. A 110:1 (2005), 127-145.

[5] A. Burstein, Enumeration of words with forbidden patterns, Ph.D. thesis, University of Pennsylvania, 1998.

[6] J. Fox, Stanley-Wilf limits are typically exponential, to appear in Adv. Math.

[7] V. Jelinek and T. Mansour, Wilf-equivalence on k-ary words, compositions, and parking functions, Elect. J. Combin. 16 (2009) \#R58.

[8] S. Kitaev, Patterns in Permutations and Words, Springer, 2011.

[9] M. Klazar, A. Marcus, Extensions of the linear bound in the FüredHajnal conjecture, Adv. in Appl. Math 38:2 (2007), 258-266.

[10] T. Mansour, R. Rastegar, A. Roitershtein, Finite automata, probabilistic method, and occurrence enumeration of a pattern in words and permutations, SIAM Disc. Math. 34:2 (2020), 1011-1038.

[11] A. Marcus, G. Tardos, Excluded permutation matrices and the StanleyWilf conjecture, J. Combin. Theory Ser. A 107:1 (2004), 153-160.

[12] A. Regev, Asymptotics of the number of k-words with an l-descent, Elect. J. Combin. 5:1 (1998), \#R15.

[13] R. Stanley, Enumerative combinatorics, Volume I, Cambridge University Press, Second Edition, 2012. 